PROCEEDINGS OF THE

AMERICAN MATHEMATICAL SOCIETY

Volume 128, Number 3, Pages 701-708

S 0002-9939(99)05619-1

Article electronically published on September 27, 1999

\title{
THETA FUNCTIONS OF INDEFINITE QUADRATIC FORMS OVER REAL NUMBER FIELDS
}

\author{
OLAV K. RICHTER
}

(Communicated by Dennis A. Hejhal)

\begin{abstract}
We define theta functions attached to indefinite quadratic forms over real number fields and prove that these theta functions are Hilbert modular forms by regarding them as specializations of symplectic theta functions. The eighth root of unity which arises under modular transformations is determined explicitly.
\end{abstract}

\section{INTRODUCTION}

We construct a theta function attached to a quadratic form over a totally real number field and show that this theta function is a modular form. If the quadratic form is not totally positive, the usual sum over some ideal in the ring of integers will not converge. Stopple [8] solves this problem by introducing the analogue of a spherical harmonic for the theta function to ensure convergence. Furthermore, he follows Eichler [2] to show that his theta function is a modular form on some $\Gamma_{0}^{0}$ subgroups. Following Siegel [5] and [6], Friedberg [3] defines a theta function of indefinite quadratic forms over $\mathbb{C}$ by using the majorants of the quadratic forms to guarantee that the theta function will converge. By converting this theta function into a symplectic theta function, Friedberg proves that his theta function is indeed a modular form on some special subgroups of $S L_{2}(\mathbb{R})$. The advantage of his method is that one can compute the theta multiplier explicitly without too much effort using the main result of Stark [7]. We will follow his method and obtain results similar to his over totally real number fields, i.e. we will prove that the theta function we will construct is a Hilbert modular form on some $\Gamma_{0}$ subgroups and also compute the theta multiplier explicitly using the main result of [7].

\section{Symplectic theta functions}

The symplectic group, $\operatorname{Sp}_{n}(\mathbb{R})$, consists of those $2 n \times 2 n$ real matrices

$$
M=\left(\begin{array}{cc}
A & B \\
C & D
\end{array}\right)
$$

(each entry is $n \times n)$ such that

$$
{ }^{t} M J M=J=\left(\begin{array}{cc}
0 & -I_{n} \\
I_{n} & 0
\end{array}\right) .
$$

Received by the editors April 29, 1998 .

1991 Mathematics Subject Classification. Primary 11F41; Secondary 11F27. 
The corresponding symmetric space is the Siegel upper half plane $\mathfrak{H}^{(n)}$ which consists of $n \times n$ symmetric complex matrices $Z$ with $\operatorname{Im}(Z)>0$ (positive definite). The action of $M$ on $Z$ is given by

$$
M \circ Z=(A Z+B)(C Z+D)^{-1} .
$$

Let $\Gamma^{(n)}=\operatorname{Sp}_{n}(\mathbb{Z})$. The theta subgroup $\Gamma_{\vartheta}^{(n)}$ of $\Gamma^{(n)}$ is the set of all $\left(\begin{array}{cc}A & B \\ D\end{array}\right)$ in $\Gamma^{(n)}$ such that both $A^{t} B$ and $C^{t} D$ have even diagonal entries. The subgroup acts on the symplectic theta function,

$$
\vartheta\left(Z,\left(\begin{array}{l}
u \\
v
\end{array}\right)\right)=\sum_{m \in \mathbb{Z}^{n}} \exp \left\{\pi i\left[{ }^{t}(m+v) Z(m+v)-2{ }^{t} m u-{ }^{t} v u\right]\right\},
$$

where $u$ and $v$ are column vectors in $\mathbb{C}^{n}$. It is well known (see Eichler [1], for example) that for

$$
\begin{gathered}
M=\left(\begin{array}{cc}
A & B \\
C & D
\end{array}\right) \text { in } \Gamma_{\vartheta}^{(n)}, \\
\vartheta\left(M \circ Z, M\left(\begin{array}{l}
u \\
v
\end{array}\right)\right)=\chi(M)[\operatorname{det}(C Z+D)]^{1 / 2} \vartheta\left(Z,\left(\begin{array}{l}
u \\
v
\end{array}\right)\right)
\end{gathered}
$$

where $\chi(M)$ is an eighth root of unity which depends upon the chosen square root of $\operatorname{det}(C Z+D)$, but which is otherwise independent of $Z, u$, and $v$. It is also known that $\chi(M)$ can be expressed in terms of Gaussian sums. Stark [7] determined $\chi(M)$ in the important special case that $p D^{-1}$ is integral for some odd prime $p$. The main result in [7] is

Theorem 1. Suppose $M=\left(\begin{array}{ll}A & B \\ C & D\end{array}\right)$ is in $\Gamma_{\vartheta}^{(n)}$ where $C^{-1}$ and $D^{-1}$ exist. Suppose further that for some odd prime $p, p D^{-1}$ is integral. Then $(\bmod p)$, the symmetric matrix $p D^{-1} C$ has rank $h$ where $\operatorname{det}(D)= \pm p^{h}$. Let $\left(p D^{-1} C\right)^{(h)}$ be a nonsingular $(\bmod p) h \times h$ principal submatrix of $p D^{-1} C$ and let $s$ be the signature (the number of positive eigenvalues minus the number of negative eigenvalues) of $C^{-1} D$. Then

$$
\begin{aligned}
& \chi(M)[\operatorname{det}(C Z+D)]^{1 / 2} \\
& =\varepsilon_{p}^{-h}\left(\frac{2^{h} \operatorname{det}\left[\left(p D^{-1} C\right)^{(h)}\right]}{p}\right) e^{\frac{\pi i s}{4}}|\operatorname{det}(C)|^{1 / 2}\left\{\operatorname{det}\left[-i C^{-1}(C Z+D)\right]\right\}^{1 / 2},
\end{aligned}
$$

where $\varepsilon_{p}=1$ for $p \equiv 1 \bmod 4, \varepsilon_{p}=i$ for $p \equiv 3 \bmod 4,(\dot{\bar{p}})$ is the Legendre symbol, $|\operatorname{det}(C)|^{1 / 2}$ is positive and $\left\{\operatorname{det}\left[-i C^{-1}(C Z+D)\right]\right\}^{1 / 2}$ is given by analytic continuation from the principal value when $Z=-C^{-1} D+i Y$. Alternatively, if just $C^{-1}$ exists and $p C^{-1}$ is integral, $\operatorname{det}(C)= \pm p^{h}$, then $p C^{-1} D(\bmod p)$ has rank $h$ and

$$
\begin{aligned}
& \chi(M)[\operatorname{det}(C Z+D)]^{1 / 2} \\
& =\varepsilon_{p}^{-h}\left(\frac{-2}{p}\right)^{h}\left(\frac{\operatorname{det}\left[\left(p C^{-1} D\right)^{(h)}\right]}{p}\right)|\operatorname{det}(C)|^{1 / 2}\left\{\operatorname{det}\left[-i C^{-1}(C Z+D)\right]\right\}^{1 / 2} .
\end{aligned}
$$




\section{ThetA FUnCtions ATTACHED TO INDEFINITE QUADRATIC FORMS}

Let $K$ be a totally real number field of degree $r_{1}$. Let $\Delta_{K}$ be the discriminant of $K$, let $\delta_{K}$ be the different of $K$, and let $\mathfrak{O}_{K}$ be the ring of integers of $K$. The algebraic conjugates of an algebraic number $\alpha$ in $K$ are given by $\alpha^{(1)}, \ldots, \alpha^{\left(r_{1}\right)}$. Furthermore, let $\Gamma=S L_{2}\left(\mathfrak{O}_{K}\right)$ and as usual, for an integral ideal $\mathfrak{N}$, let

$$
\Gamma_{0}(\mathfrak{N})=\left\{M=\left(\begin{array}{ll}
\alpha & \beta \\
\gamma & \delta
\end{array}\right), M \in \Gamma \text { and } \gamma \in \mathfrak{N}\right\} .
$$

We define the upper half plane $\mathfrak{H}=\mathbb{H}^{r_{1}}$, where $\mathbb{H}=\{z \in \mathbb{C}, \operatorname{Im} z>0\}$ is the usual upper half plane. The matrix

$$
M=\left(\begin{array}{cc}
\alpha & \beta \\
\gamma & \delta
\end{array}\right) \in S L_{2}(K)
$$

acts on $z=\left(z_{1}, \ldots, z_{r_{1}}\right) \in \mathfrak{H}$ by

$$
M \circ z=\left(M^{(1)} \circ z_{1}, \ldots, M^{\left(r_{1}\right)} \circ z_{r_{1}}\right),
$$

where

$$
M^{(j)}=\left(\begin{array}{ll}
\alpha^{(j)} & \beta^{(j)} \\
\gamma^{(j)} & \delta^{(j)}
\end{array}\right)
$$

and

$$
M^{(j)} \circ z_{j}=\left(\alpha^{(j)} z_{j}+\beta^{(j)}\right)\left(\gamma^{(j)} z_{j}+\delta^{(j)}\right)^{-1} .
$$

For $\gamma$ and $\delta$ in $K$ and $z$ in $\mathfrak{H}$, we define

$$
\mathcal{N}(\gamma z+\delta)=\prod_{j=1}^{r_{1}}\left(\gamma^{(j)} z_{j}+\delta^{(j)}\right)
$$

and

$$
\mathcal{N}\left[(\gamma z+\delta)^{1 / 2}\right]=\prod_{j=1}^{r_{1}}\left(\gamma^{(j)} z_{j}+\delta^{(j)}\right)^{1 / 2},
$$

where each of the $r_{1}$ square roots is given by the principal value.

Let $Q$ be a symmetric $n \times n$ matrix defining the quadratic form $Q[x]={ }^{t} x Q x$, where $x \in \mathbb{R}^{n}$. If $Q$ has entries in $\mathfrak{O}_{K}$ and diagonal entries which are divisible by 2, we say that $Q$ is of level $N\left(N \in \mathfrak{O}_{K}\right)$ whenever the following two conditions are satisfied:

a) The matrix $N Q^{-1}$ has entries in $\mathfrak{O}_{K}$, and 2 divides the diagonal entries of $N Q^{-1}$.

b) For any $M \in \mathfrak{O}_{K}, N$ divides $M$ whenever $M Q^{-1}$ has entries in $\mathfrak{O}_{K}$ and 2 divides the diagonal entries of $M Q^{-1}$.

If $Q^{(j)}$ has signature $(p, q)$ for $j=1, \ldots, r_{1}$, then there exist matrices $L_{j}$ in $G L_{n}(\mathbb{R})$ such that $Q^{(j)}={ }^{t} L_{j} E_{p, q} L_{j}$, where

$$
E_{p, q}=\left(\begin{array}{cc}
I_{p} & \\
& -I_{q}
\end{array}\right),
$$

and $I_{p}$ and $I_{q}$ are the $p \times p$ and $q \times q$ identity matrices, respectively.

Set $R_{j}={ }^{t} L_{j} L_{j}$. Then $R_{j}$ is a majorant of $Q^{(j)}$, i.e.

$$
R_{j} Q^{(j)^{-1}} R_{j}=Q^{(j)} \text { and }{ }^{t} R_{j}=R_{j}>0 .
$$


For the vector $\lambda={ }^{t}\left(\lambda_{1}, \ldots, \lambda_{n}\right)$, we set $\lambda^{(j)}={ }^{t}\left(\lambda_{1}^{(j)}, \ldots, \lambda_{n}^{(j)}\right)$ where $\lambda_{1}, \ldots, \lambda_{n}$ are in $K$. We define the theta function $\Theta_{Q}$ of an indefinite quadratic form by

Definition 1. Let $Q$ be a symmetric $n \times n$ matrix with entries in $\mathfrak{O}_{K}$ such that 2 divides the diagonal entries of $Q$ and such that $Q$ is of level $N$. Furthermore, assume that each $Q^{(j)}$ has the same signature $(p, q)$ for $j=1, \ldots, r_{1}$. Let $u_{1}, \ldots, u_{r_{1}}$ and $v_{1}, \ldots, v_{r_{1}}$ be vectors in $\mathbb{C}^{n}$. For an ideal $\mathfrak{I} \subset \mathfrak{O}_{K}$ and $z=\left(z_{1}, \ldots, z_{r_{1}}\right) \in \mathfrak{H}$ set

$$
\begin{aligned}
\Theta_{Q}\left(z,\left(\begin{array}{c}
u \\
v
\end{array}\right)\right) & =\left(\prod_{j=1}^{r_{1}} y_{j}\right)^{q / 2} \sum_{\lambda \in \mathfrak{I}^{n}} \exp \left\{\pi i \left[\sum_{j=1}^{r_{1}} Q^{(j)}\left[\lambda^{(j)}+v_{j}\right] x_{j}\right.\right. \\
& \left.\left.+i R_{j}\left[\lambda^{(j)}+v_{j}\right] y_{j}-2{ }^{t} \lambda^{(j)} Q^{(j)} u_{j}-{ }^{t} v_{j} Q^{(j)} u_{j}\right]\right\},
\end{aligned}
$$

where $\lambda={ }^{t}\left(\lambda_{1}, \ldots, \lambda_{n}\right), u={ }^{t}\left({ }^{t} u_{1}, \ldots,{ }^{t} u_{r_{1}}\right)$ and $v={ }^{t}\left({ }^{t} v_{1}, \ldots,{ }^{t} v_{r_{1}}\right)$.

Note that for any algebraic integer $t \in K, \sum_{j=1}^{r_{1}} Q^{(j)}\left[\lambda^{(j)}\right] t^{(j)}=\operatorname{tr}(Q[\lambda] t)$ is an even rational integer, and thus $\Theta_{Q}(z)$ is invariant under linear transformations, i.e.

$$
\Theta_{Q}\left(z+t,\left(\begin{array}{c}
u+t v \\
v
\end{array}\right)\right)=\Theta_{Q}\left(z,\left(\begin{array}{l}
u \\
v
\end{array}\right)\right) .
$$

The first task is to convert $\Theta_{Q}$ into a symplectic theta function. Let $\omega_{1}, \ldots, \omega_{r_{1}}$ be an integral basis of the ideal $\mathfrak{I} \subset \mathfrak{O}_{K}$ and define the vector $\omega^{(j)}=\left(\omega_{1}^{(j)}, \ldots, \omega_{r_{1}}^{(j)}\right)$. We define the $n \times n r_{1}$ matrix

$$
W_{j}=\left(\begin{array}{ccc}
\omega^{(j)} & & \\
& \ddots & \\
& & \omega^{(j)}
\end{array}\right)
$$

and the $n r_{1} \times n r_{1}$ matrix $W={ }^{t}\left({ }^{t} W_{1}, \ldots,{ }^{t} W_{r_{1}}\right)$. Note that $W^{-1}$ has entries in $\mathfrak{I}^{-1} \delta_{K}^{-1}$.

For $z=\left(z_{1}, \ldots, z_{r_{1}}\right) \in \mathfrak{H}$, set

$$
Z_{j}^{*}=\left(\begin{array}{cc}
z_{j} I_{p} & \\
& -\overline{z_{j}} I_{q}
\end{array}\right)
$$

and

$$
Z^{*}=\left(\begin{array}{ccc}
Z_{1}^{*} & & \\
& \ddots & \\
& & Z_{r_{1}}^{*}
\end{array}\right),
$$

where $\overline{z_{j}}$ is the complex conjugate of $z_{j}$. Define

$$
L=\left(\begin{array}{ccc}
L_{1} & & \\
& \ddots & \\
& & L_{r_{1}}
\end{array}\right)
$$

and set

$$
T=L W \text { and } Z={ }^{t} T Z^{*} T=\left(\begin{array}{cc}
{ }^{t} T & 0 \\
0 & T^{-1}
\end{array}\right) \circ Z^{*}
$$


Observe that $\left(\begin{array}{cc}{ }^{t} T & 0 \\ 0 & T^{-1}\end{array}\right)$ is in $\operatorname{Sp}_{n r_{1}}(\mathbb{R})$. Furthermore, $Z^{*}$ is in the Siegel upper half plane $\mathfrak{H}^{\left(n r_{1}\right)}$ and therefore $Z$ is in $\mathfrak{H}^{\left(n r_{1}\right)}$ as well. We have

$$
\left(\prod_{j=1}^{r_{1}} y_{j}\right)^{-q / 2} \Theta_{Q}\left(z,\left(\begin{array}{c}
u \\
v
\end{array}\right)\right)=\vartheta\left(Z,\left(\begin{array}{c}
{ }^{t} W \widetilde{Q} u \\
W^{-1} v
\end{array}\right)\right),
$$

where

$$
\widetilde{Q}=\left(\begin{array}{ccc}
Q^{(1)} & & \\
& \ddots & \\
& & Q^{\left(r_{1}\right)}
\end{array}\right)
$$

In order to apply Theorem 1, we need a symplectic matrix which expresses the action of $\left(\begin{array}{ll}\alpha & \beta \\ \gamma & \delta\end{array}\right) \in \Gamma=S L_{2}\left(\mathfrak{O}_{k}\right)$ on our new variables $u, v$ and $Z$. For $\left(\begin{array}{ll}\alpha & \beta \\ \gamma & \delta\end{array}\right) \in \Gamma$, set

$$
\left(\begin{array}{cc}
A^{*} & B^{*} \\
C^{*} & D^{*}
\end{array}\right)=\left(\begin{array}{cccccc}
\alpha^{(1)} I_{n} & & & \beta^{(1)} E_{p, q} & & \\
& \ddots & & & \ddots & \\
& & \alpha^{\left(r_{1}\right)} I_{n} & & & \beta^{\left(r_{1}\right)} E_{p, q} \\
& & \delta^{(1)} I_{n} & & \\
\gamma^{(1)} E_{p, q} & & & & \ddots & \\
& \ddots & & & & \delta^{\left(r_{1}\right)} I_{n}
\end{array}\right)
$$

It is easy to check that the diagrams

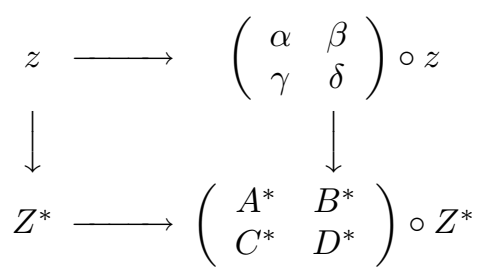

and

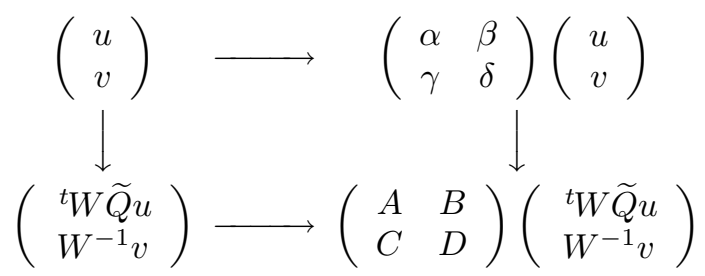

commute, where

$$
\begin{aligned}
\left(\begin{array}{ll}
A & B \\
C & D
\end{array}\right) & =\left(\begin{array}{cc}
{ }^{t} T & 0 \\
0 & T^{-1}
\end{array}\right)\left(\begin{array}{cc}
A^{*} & B^{*} \\
C^{*} & D^{*}
\end{array}\right)\left(\begin{array}{cc}
{ }^{t} T & 0 \\
0 & T^{-1}
\end{array}\right)^{-1} \\
& =\left(\begin{array}{cc}
{ }^{t} T A^{*} T^{-1} & { }^{t} T B^{*} T \\
{ }^{T}{ }^{-1} C^{*} T^{-1} & T^{-1} D^{*} T
\end{array}\right)
\end{aligned}
$$

Hence

$$
z \mapsto\left(\begin{array}{cc}
\alpha & \beta \\
\gamma & \delta
\end{array}\right) \circ z
$$


in $\mathfrak{H}$ corresponds to

$$
Z \mapsto\left(\begin{array}{cc}
A & B \\
C & D
\end{array}\right) \circ Z
$$

in $\mathfrak{H}^{\left(n r_{1}\right)}$.

Let us introduce some more notation to show conditions under which the matrix in (7) is in the theta subgroup. Assume that $S=\left(s_{i l}\right)_{i, l=1, \ldots, r_{1}}$ and $R=$ $\left(r_{k m}\right)_{k, m=1, \ldots, n}$ are matrices with entries in $K$. We define the matrix $R \odot S=$ $\left(\left(\operatorname{tr}\left(r_{k m} s_{i l}\right)_{i, l=1, \ldots, r_{1}}\right)\right)_{k, m=1, \ldots, n}$. Note that the entries of $R \odot S$ are rational numbers. Computation shows that $A=I_{n} \odot A^{\prime}, B=Q \odot B^{\prime}, C=Q^{-1} \odot C^{\prime}$ and $D=I_{n} \odot D^{\prime}$, where $A^{\prime}, B^{\prime}, C^{\prime}$ and $D^{\prime}$ are given by

$$
\begin{aligned}
A^{\prime} & =\left(\begin{array}{ccc}
\omega_{1} \nu_{1} \alpha & \cdots & \omega_{1} \nu_{r_{1}} \alpha \\
\vdots & & \vdots \\
\omega_{r_{1}} \nu_{1} \alpha & \cdots & \omega_{r_{1}} \nu_{r_{1}} \alpha
\end{array}\right), \\
B^{\prime} & =\left(\begin{array}{ccc}
\omega_{1} \omega_{1} \beta & \cdots & \omega_{1} \omega_{r_{1}} \beta \\
\vdots & & \vdots \\
\omega_{r_{1}} \omega_{1} \beta & \cdots & \omega_{r_{1}} \omega_{r_{1}} \beta
\end{array}\right), \\
C^{\prime} & =\left(\begin{array}{ccc}
\nu_{1} \nu_{1} \gamma & \cdots & \nu_{1} \nu_{r_{1}} \gamma \\
\vdots & & \vdots \\
\nu_{r_{1}} \nu_{1} \gamma & \cdots & \nu_{r_{1}} \nu_{r_{1}} \gamma
\end{array}\right), \\
D^{\prime} & =\left(\begin{array}{ccc}
\omega_{1} \nu_{1} \delta & \cdots & \omega_{r_{1}} \nu_{1} \delta \\
\vdots & & \vdots \\
\omega_{1} \nu_{r_{1}} \delta & \cdots & \omega_{r_{1}} \nu_{r_{1}} \delta
\end{array}\right) .
\end{aligned}
$$

Clearly, ${ }^{t} A C={ }^{t} C A,{ }^{t} B D={ }^{t} D B$, and ${ }^{t} D A-{ }^{t} B C=I_{n r_{1}}$. Hence $\left(\begin{array}{l}A \\ C\end{array}\right.$ $\mathrm{Sp}_{n r_{1}}(\mathbb{R})$. Furthermore, $A^{t} B={ }^{t} T A^{*} B^{*} T=Q \odot\left(\alpha B^{\prime}\right)$ and $C^{t} D=T^{-1} C^{*} D^{* t} T^{-1}$ $=Q^{-1} \odot\left(\delta C^{\prime}\right)$. It follows that the entries of $A, B, C$ and $D$ are rational integers and that $A^{t} B$ and $C^{t} D$ have even diagonal entries if $\gamma$ is in the ideal $\mathfrak{I}^{2} \delta_{K} N$. Hence for

$$
\left(\begin{array}{cc}
\alpha & \beta \\
\gamma & \delta
\end{array}\right) \in \Gamma_{0}\left(\Im^{2} \delta_{K} N\right)
$$

we have

$$
\left(\begin{array}{cc}
A & B \\
C & D
\end{array}\right) \in \Gamma_{\vartheta}^{\left(n r_{1}\right)}
$$

It is easy to verify that

$$
\operatorname{det}(C Z+D)=\operatorname{det}\left(C^{*} Z^{*}+D^{*}\right)=\mathcal{N}(\gamma z+\delta)^{p} \mathcal{N}(\gamma \bar{z}+\delta)^{q},
$$

and therefore by equations (1) and (5),

$$
\begin{aligned}
& \Theta_{Q}\left(\left(\begin{array}{ll}
\alpha & \beta \\
\gamma & \delta
\end{array}\right) \circ z,\left(\begin{array}{ll}
\alpha & \beta \\
\gamma & \delta
\end{array}\right)\left(\begin{array}{l}
u \\
v
\end{array}\right)\right) \\
& =\chi\left(\left(\begin{array}{ll}
\alpha & \beta \\
\gamma & \delta
\end{array}\right), Q\right) \mathcal{N}(\gamma z+\delta)^{(p-q) / 2} \Theta_{Q}\left(z,\left(\begin{array}{l}
u \\
v
\end{array}\right)\right),
\end{aligned}
$$


where $\chi\left(\left(\begin{array}{ll}\alpha & \beta \\ \gamma & \delta\end{array}\right), Q\right)$ is an eighth root of unity depending on $\left(\begin{array}{ll}\alpha & \beta \\ \gamma & \delta\end{array}\right)$ and $Q$. Thus, $\Theta_{Q}\left(z,\left(\begin{array}{l}u \\ v\end{array}\right)\right)$ is a (nonanalytic) Hilbert modular form on $\Gamma_{0}\left(\mathfrak{I}^{2} \delta_{K} N\right)$ of weight $(p-q) / 2$.

\section{The EIGHTh ROOT OF UNITY}

Now we determine $\chi\left(\left(\begin{array}{cc}\alpha & \beta \\ \gamma & \delta\end{array}\right), Q\right)$. Let us assume that $\delta \gg 0$ is a first degree prime in $\mathfrak{O}_{k}$ of norm $d$, where $d$ is a positive odd prime in $\mathbb{Z}$. In this case, $d D^{-1}$ is integral. Note that $\operatorname{det}(D)=\operatorname{det}\left(D^{*}\right)=d^{n}$, and thus by Theorem $1, d D^{-1} C$ has rank $n$ $(\bmod d)$. Hence for $Q^{-1}=\left(r_{i l}\right)_{i, l=1, \ldots, n}$, we see that

$$
\left(d D^{-1} C\right)^{(n)}=\left(\begin{array}{ccc}
\operatorname{tr}\left(r_{11} \nu_{1} \nu_{1} d \delta^{-1} \gamma\right) & \cdots & \operatorname{tr}\left(r_{1 n} \nu_{1} \nu_{1} d \delta^{-1} \gamma\right) \\
\vdots & & \vdots \\
\operatorname{tr}\left(r_{1 n} \nu_{1} \nu_{1} d \delta^{-1} \gamma\right) & \cdots & \operatorname{tr}\left(r_{n n} \nu_{1} \nu_{1} d \delta^{-1} \gamma\right)
\end{array}\right)
$$

and

$$
\operatorname{det}\left(d D^{-1} C\right)^{(n)} \equiv\left(d \delta^{-1} \gamma\right)^{n}\left(\nu_{1} \nu_{1}\right)^{n} \operatorname{det}(Q)^{-1}(\bmod \delta) .
$$

Some computation shows that

$$
|\operatorname{det}(C)|^{1 / 2}\left\{\operatorname{det}\left[-i C^{-1}(C Z+D)\right]\right\}^{1 / 2} e^{\frac{\pi i s}{4}}=\mathcal{N}(\gamma z+\delta)^{(p-q) / 2}|\mathcal{N}(\gamma z+\delta)|^{q} .
$$

Hence

$$
\chi\left(\left(\begin{array}{ll}
\alpha & \beta \\
\gamma & \delta
\end{array}\right), Q\right)=\varepsilon_{d}^{-n}\left(\frac{\left(d \delta^{-1} 2 \gamma\right)^{n} \operatorname{det}(Q)}{\delta}\right) .
$$

We have proved

Theorem 2. Suppose that $\left(\begin{array}{cc}\alpha & \beta \\ \gamma & \delta\end{array}\right) \in \Gamma_{0}\left(\mathfrak{I}^{2} \delta_{K} N\right)$, where $\delta$ is a first degree prime in $\mathfrak{O}_{K}$ of norm $d$ ( $d$ is a positive odd prime in $\mathbb{Z}$ ). For $z \in \mathfrak{H}$, we have

$$
\begin{aligned}
& \Theta_{Q}\left(\left(\begin{array}{ll}
\alpha & \beta \\
\gamma & \delta
\end{array}\right) \circ z,\left(\begin{array}{ll}
\alpha & \beta \\
\gamma & \delta
\end{array}\right)\left(\begin{array}{l}
u \\
v
\end{array}\right)\right) \\
& =\varepsilon_{d}^{-n}\left(\frac{\left(d \delta^{-1} 2 \gamma\right)^{n} \operatorname{det}(Q)}{\delta}\right) \mathcal{N}(\gamma z+\delta)^{(p-q) / 2} \Theta_{Q}\left(z,\left(\begin{array}{c}
u \\
v
\end{array}\right)\right),
\end{aligned}
$$

where $\varepsilon_{d}=1$ for $d \equiv 1 \bmod 4$ and $\varepsilon_{d}=i$ for $d \equiv 3 \bmod 4$.

Actually, we have determined the eighth root of unity more explicitly than it seems. In (4), we showed that for all algebraic integers $t$,

$$
\Theta_{Q}\left(\left(\begin{array}{ll}
1 & t \\
0 & 1
\end{array}\right) \circ z,\left(\begin{array}{ll}
1 & t \\
0 & 1
\end{array}\right)\left(\begin{array}{l}
u \\
v
\end{array}\right)\right)=\Theta_{Q}\left(z,\left(\begin{array}{l}
u \\
v
\end{array}\right)\right) .
$$

Together with (8) this implies that for $\left(\begin{array}{cc}\alpha & \beta \\ \gamma & \delta\end{array}\right) \in \Gamma_{0}\left(\mathfrak{I}^{2} \delta_{K} N\right)$ and for all algebraic integers $t$,

$$
\chi\left(\left(\begin{array}{cc}
\alpha & \beta \\
\gamma & \delta
\end{array}\right)\left(\begin{array}{ll}
1 & t \\
0 & 1
\end{array}\right), Q\right)=\chi\left(\left(\begin{array}{ll}
\alpha & \beta \\
\gamma & \delta
\end{array}\right), Q\right) .
$$

Furthermore, Hecke [4] gives a proof of Dirichlet's primes in progression theorem for number fields. Hence for algebraic integers $\gamma$ and $\delta$ with $(\gamma, \delta)=1$, the arithmetic progression $\{\gamma t+\delta\}_{t \in \mathfrak{O}_{k}}$ contains infinitely many primes $\pi \gg 0$ such that $\mathcal{N}(\pi)$ is a positive odd prime in $\mathbb{Z}$. Hence the theta multiplier is determined explicitly after locating a totally positive first degree prime in the arithmetic progression $\{\gamma t+\delta\}_{t \in \mathfrak{O}_{k}}$. 
There is a special case which also should be mentioned. Let $\delta \gg 0$ be a prime in $\mathfrak{O}_{K}$ with $\mathcal{N}(\delta)=d^{r_{1}}$, where $d$ is a positive odd prime in $\mathbb{Z}$. As before, we observe that $d D^{-1}$ has rational integers as entries and hence $D$ is of level $d$. We see that $\operatorname{det}(D)=\operatorname{det}\left(D^{*}\right)=d^{n r_{1}}$ and by Theorem $1, d D^{-1} C$ has rank $n r_{1}(\bmod d)$. Thus,

$$
\operatorname{det}\left(d D^{-1} C\right)=(\mathcal{N}(\operatorname{det}(Q)))^{-1}\left(\Delta_{K}\left(\mathcal{N}(\mathcal{I})^{2}\right)\right)^{-n}\left(\mathcal{N}\left(\gamma^{n}\right)\right) \text {. }
$$

Hence

$$
\chi\left(\left(\begin{array}{ll}
\alpha & \beta \\
\gamma & \delta
\end{array}\right), Q\right)=\varepsilon_{d}^{-n r_{1}}\left(\frac{\left(\Delta_{K}\right)^{n} \mathcal{N}\left((2 \gamma)^{n} \operatorname{det}(Q)\right)}{d}\right) .
$$

In the case that $n$ is even, we should see that (9) and (12) yield the same result. For an odd rational prime $d$, an element $a$ is a square in $\mathbb{F}_{d^{r_{1}}}$ (the field of $d^{r_{1}}$ elements) iff $\mathcal{N}_{\mathbb{F}_{d} r_{1} / \mathbb{F}_{d}}(a)$ is a square in $\mathbb{F}_{d}$ (the field of $d$ elements). This can be seen by observing that the mapping $N: \mathbb{F}_{d^{r_{1}}}^{*} \rightarrow \mathbb{F}_{d}^{*}$ given by $a \rightarrow N(a):=\mathcal{N}_{\mathbb{F}_{d^{r_{1}}} / \mathbb{F}_{d}}(a)$ is an epimorphism. Hence in the special case that $n$ is even, (9) becomes

$$
\chi\left(\left(\begin{array}{ll}
\alpha & \beta \\
\gamma & \delta
\end{array}\right), Q\right)=\left(\frac{(-1)^{n / 2} \operatorname{det}(Q)}{\delta}\right)
$$

and (12) becomes

$$
\chi\left(\left(\begin{array}{cc}
\alpha & \beta \\
\gamma & \delta
\end{array}\right), Q\right)=\left(\frac{\mathcal{N}\left((-1)^{n / 2} \operatorname{det}(Q)\right)}{d}\right),
$$

and the result from (9) coincides with the result from (12).

\section{REFERENCES}

[1] M. Eichler, Introduction to the theory of algebraic numbers and functions, Academic Press, New York, 1966. MR 35:160

[2] _ On theta functions of real algebraic number fields, Acta Arithmetica 33 (1977), 269292. MR 58:27788

[3] S. Friedberg, On theta functions associated to indefinite quadratic forms, Journal of number theory 23 (1986), 255-267. MR 87k:11051

[4] E. Hecke, Über die L-Funktionen und den Dirichletschen Primzahlsatz für einen beliebigen Zahlkörper, Nachrichten der K. Gesellschaft der Wissenschaften zu Göttingen, Mathematischphysikalische Klasse (1917), 299-318.

[5] C. Siegel, Indefinite Quadratische Formen und Funktionentheorie I, Math. Ann. 124 (1951), 17-54. MR 16:800a

[6] - Indefinite Quadratische Formen und Funktionentheorie II, Math. Ann. 124 (1952), 364-387. MR 16:801a

[7] H. Stark, On the transformation formula for the symplectic theta function and applications, J. Fac. of Sci. Univ. of Tokyo, Section 1A, 29 (1982), 1-12. MR 83j:10029

[8] J. Stopple, Theta series for indefinite quadratic forms over real number fields, Acta Arithmetica 72 (1995), 299-309. MR 97b:11061

Department of Mathematics, University of California, San Diego, California 920930112

E-mail address: richter@euclid.ucsd.edu

Current address: Department of Mathematics, University of California, Santa Cruz, California 95064

E-mail address: richter@math.ucsc.edu 